\title{
The Fault Check and Analysis of High Pressure Water Valve in Water Isolated Slurry Pump Shaoyi Wang ${ }^{1 \mathrm{a}}$, Ping Sun ${ }^{2 \mathrm{~b}}$, Yang Lic ${ }^{1 \mathrm{c}}$, Kangkang Zhou ${ }^{1 \mathrm{~d}}$
}

1. College of Mechanical Engineering, Shenyang University, Liaoning, 110044, China

2. SYU-Slurry Transportation Research Institute, Liaoning, 110044, China

a857618921@qq.com, bssunpp@263.net, '836885272@qq.com, ${ }^{\mathrm{d} z k 9708 @ 163 . c o m}$

Keywords: Water isolated slurry pump, High pressure water valve, Pressure on the entrance and exit point, Fault diagnosis and analysis.

\begin{abstract}
Currently, water isolated slurry pump has been widely used in non-ferrous metal mines, which to replace the low-lift pump as a new water isolated slurry pump. The water isolated slurry pump consists of high pressure water pump, isolation devices, check valves, water valves, control systems, auxiliary devices. The high pressure water valve is a high wearing part as one of controlled valve. By setting pressure pickups on the entrance and exit point, detecting the pressure data and wave. So we can diagnose whether the high pressure water valve is wrong and analyze it. By using this method, the equipment maintainer can quickly make a judgment. And the water isolated slurry pump can be operated normally.
\end{abstract}

\section{Introduction}

The slurry pipeline transportation usually uses the water as a carrier and transports the granular materials by the way of pipeline ${ }^{[1]}$. As the fifth mode of transportation in transport system, compared to other modes of transportation, with less investment, short construction cycle, low energy consumption, low transportation costs and the advantage of continuous operation. And now the slurry pipeline has been widely used in the transportation of concentrate and tailing heap of non-metal and metal mines, red mud of aluminum plant, mud of water conservancy ${ }^{[2]}$, etc. The high pressure water valve is one of the most important control valves in the use of water isolated slurry pump in the long distance slurry pipeline transportation in china. Because the water isolated slurry pump require cyclical supply and discharge the slurry. The high pressure water valve in water isolated slurry pump and the isolated canister both work under the alternating pressure. And the high pressure water valve open and close frequently. It is a high wearing part as one of controlled valve in water isolated slurry pump. Once the fault appears, and we can not find it timely, the fault will lead to a serious consequence about the run of the system's security. Of course, the productivity will be lower ${ }^{[3]}$. The main fault of high pressure water valve in water isolated slurry pump include of valve sticking, valve fracture and valve wear. In order to determine promptly whether the high pressure water valve in water isolated slurry pump was wrong, we set pressure pickups on the entrance and exit point, detecting the pressure data and wave. So we can diagnose whether the high pressure water valve is wrong and analyze it in this text.

\section{The system of water isolated slurry pump}

\section{The working principle of water isolated slurry pump system}

The water isolated slurry pump use the water as the driving fluid and transmission media, and use the high pressure centrifugal water pump as the power source ${ }^{[4]}$. The working principle, as is shown in Fig. 1. 


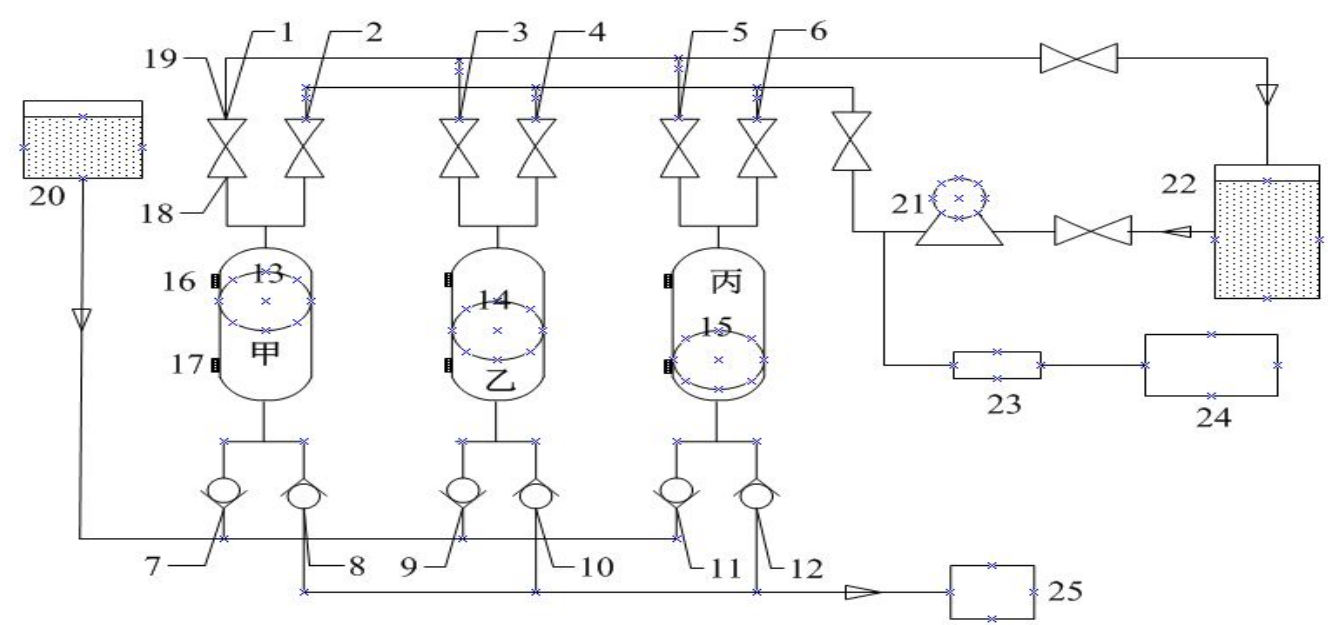

Fig. 1 The working principle of water isolated slurry pump system

1-6-high pressure water valve, 7-12- check valve, 13-15-isolated ball, 16-17-the detection of pipeline pressure device, 18-19- pressure pickups, 20- slurry Warehouse, 21- centrifugal pump, 22-water tank, 23- hydraulic Station, 24- control system of microcomputer , 25- tailing heap

In the water isolated slurry pump, the slurry Warehouse 20 provide slurry to the bottom of floating ball in the three isolated tanks of class A, B, C. while we use the high pressure water pump provide water to the top of floating ball in the three isolated tanks of class $\mathrm{A}, \mathrm{B}, \mathrm{C}$. The water drives the high pressure to the slurry through the floating ball 13-15 by using the power of water. And the slurry will be transported to the specified location through the slurry pipeline. The hydraulic Station 23 under the control of system of microcomputer 24 drive six high pressure water valves 1-6 to open and close alternately, and it can ensure class of A, B, C cyclically supply and discharge the slurry. So it can continuously transport the slurry. And we can use the system of microcomputer to monitor real-time signal by using the detection of pipeline pressure device 16-17, and we can diagnose whether the high pressure water valve is wrong and analyze it. The pressure of the high pressure water pump is great when it provide the water to the isolated tanks. What is more, the high-pressure water valve has been turned on and off frequently. So its vulnerable wearing rate is high. Thus it is critical that we can diagnose whether the high pressure water valve is wrong. The water isolated slurry pump work normally only In the case of timely discovery.

\section{The working principle of one water isolated slurry pump}

The slurry Warehouse 20 provide slurry to the isolated tanks of class $C$ through the check valve 14, and the floating ball 7 move up under the high pressure. The back-water valve 2 opens so that the water on the top of the floating ball 7 goes back to the water tank 9. When the isolated ball 7 arrive to the detection, which pipeline pressure device on the top of the isolated tank, the back-water valve of 2 closes. But the inlet valve 1 open and the floating ball 7 move down under the high pressure. At the moment, the check valve 14 close, but the check valve 15 open. It can transport the tailing heap slurry to the tailing heap. When the isolated ball 7 arrive to the detection of pipeline pressure device on the bottom of the isolated tank, the back-water valve 2 open and the inlet valve 1 close. Hence one single process of cyclical supply and discharge the slurry is completed. It's working principle, as is shown in Fig. 2. 


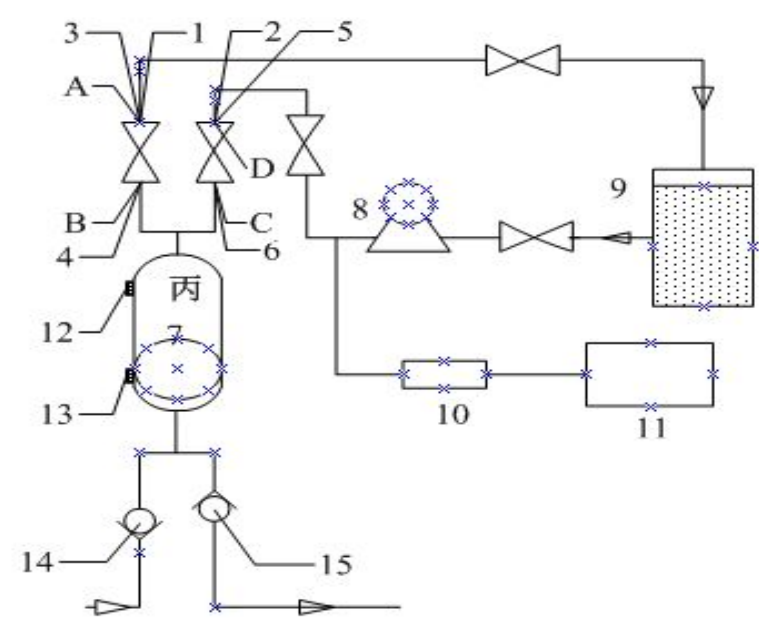

Fig. 2 The working principle of one water isolated slurry pump

1-2- high pressure water valve, 3-6- pressure pickups, 7- isolated ball, 8- centrifugal pump,9-water tank, 10-hydraulic Station, 11-control system of microcomputer ,12-13- the detection of pipeline pressure device, $14-15$ - check valve

\section{The structure and fault check of high pressure water valve}

The structure of the high pressure water valve ${ }^{[5]}$ in Fig. 3

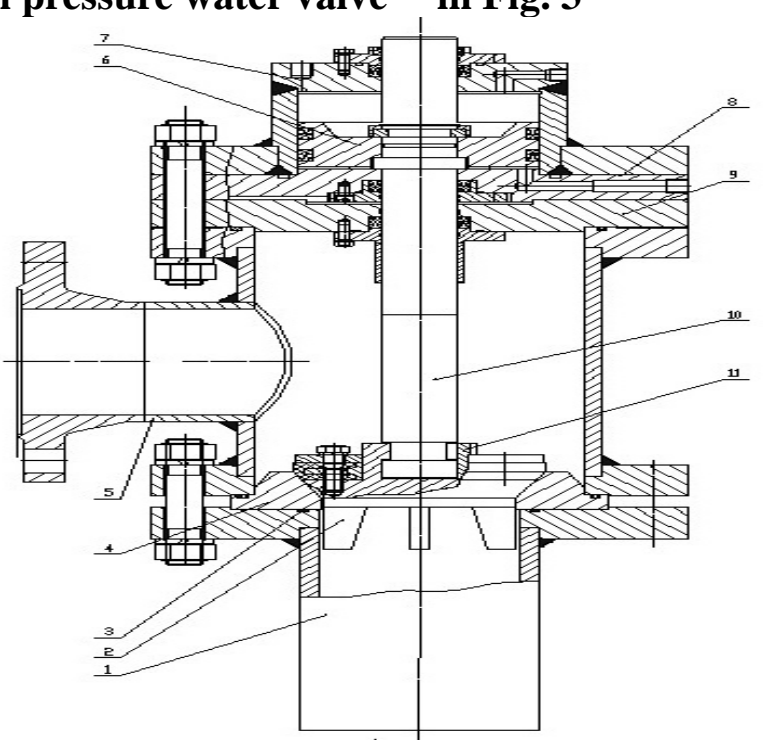

Fig. 3 The structure of the high pressure water valve

1-the top of valve body, 2-valve plug, 3- Seal Ring, 4- valve seat, 5-the bottom of valve body, 6piston, 7- hydro-cylinder, 8- hydraulic cylinder head, 9-valve head, 10-stem, 11-block

\section{The fault of the high pressure water valve}

As is shown in Fig.3, the water isolated pump requires cyclical supply and discharge the slurry. At the same time, the high pressure water valve in water isolated slurry pump and isolated tank both work under the mutative pressure. And the high pressure water valve in water isolated slurry pump needs often open and close. So the high pressure water valve always wears and tears. For the large-scale mining, it is a very serious problem that the leak problem once happens. What is the important is that it pollutes our environment. So it is necessary to solve the problem of the fault of the high pressure water valve.

\section{The fault diagnosis mechanism of the high pressure water valve}

The diagnostic methods of the problem of the wearing valve plug about the high pressure water valves are diverse. In order to be able to promptly diagnose whether the high pressure water valve is wrong and analyze it, we set pressure pickups 3-6 on the entrance and exit point A, B, C, D to detect the pressure data and wave ${ }^{[6]}$ in this text. 
Using this method, we can quickly determine and judge the fault point of the high pressure water valve according to the Number Signal and audible alarm. It is quite clear that it is a useful method to quickly determine whether the valve plug wrong. Certainly, it improves the inspection efficiency [7].

\section{The fault diagnostic process of the high pressure water valve}

By setting pressure pickups 3, 4, 5, 6 on the entrance and exit point $\mathrm{A}, \mathrm{B}, \mathrm{C}, \mathrm{D}$. We can use the procedures previously entered in the computer control system to analyze the pressure fluctuation signals. And we use the PC's text prompts and the alarm to determine whether the high pressure water valve is wrong.

\section{The pressure detection process of discharging the slurry}

In Figure 4, we take the tank $C$ for example to detect the fault of the high pressure water valve. Because the detection principle of A, B, C is same, so we just detect the tank C. In the process of discharging the slurry, the faults of the high pressure water valve include the inlet valve's fault and the back-water valve's fault.

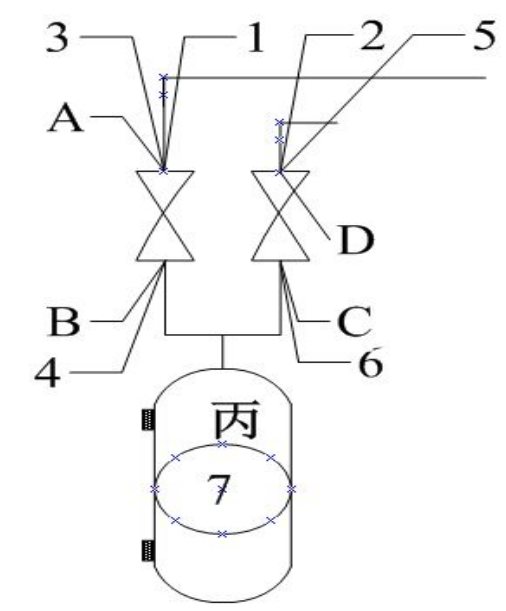

Fig. 4 The pressure detection of the tank $\mathrm{C}$

1-2- high pressure water valve, 3-6- pressure pickups, 7- isolated ball.

\section{The check and analysis of the first fault}

The first fault: The flow of supplying the slurry is less than the predetermined flow ${ }^{[8]}$ of supplying the slurry, and the most important thing is that the system pressure is high when it supplies the slurry. But the system pressure is low when it discharges the slurry. So we can determine that the high pressure water valve 1 is wrong. It includes three specific phenomenons.

(1) The measured pressure value of the inlet valve $1 \mathrm{~A}$ is low than the normal value, and the measured pressure value of the inlet valve $1 \mathrm{~B}$ is high than the normal value. But the pressure value of the inlet valve $1 \mathrm{~A}$ and the inlet valve $1 \mathrm{~B}$ is not equal.

(2) The measured pressure value of the inlet valve $1 \mathrm{~A}$ is equal to the pressure value of the inlet valve $1 \mathrm{~B}$.

(3) The measured pressure value of the inlet valve $1 \mathrm{~A}$ is high than the pressure value of the inlet valve $1 \mathrm{~B}$.

\section{The check and analysis of the first fault:}

The reason for the first phenomenon is that the high pressure water valve 1 may be wrong. The main reasons: there are large particles attached between valve plug and valve seat, so they scratch the sealing surface. It also is the case that probably the wearing valve plug make the valve plug and valve seat failure to strictly sealed. 
The reason for the second phenomenon is that the high pressure water valve 1 is wrong. The main reasons: The high pressure water driving force ${ }^{[9]}$ is so large that the high pressure water valve is fully open. So the pressure value of the inlet valve $1 \mathrm{~A}$ is equal to the pressure value of the inlet valve $1 \mathrm{~B}$.

The reason for the third phenomenon is that the high pressure water valve 1 meet to the requirements, and it works well.

\section{The check and analysis of the second fault}

The second fault: The flow of supplying the slurry is more than the predetermined flow of supplying the slurry, and the most important thing is that the system pressure is high when it supplies the slurry. But the system pressure is low when it discharges the slurry. So we can judge that the high pressure water valve 2 is wrong.

Its specific phenomenon is that the measured pressure value of the back-water valve $2 \mathrm{C}$ is not equal to the pressure value of the inlet valve $2 \mathrm{D}$.

\section{The check and analysis of the second fault:}

The reason for this phenomenon is that the high pressure back-water valve's stem is wrong when it works under the high pressure water driving force. The other reason for this phenomenon is that the guide sleeve is wrong.

Because one single experiment of cyclical supply and discharge the slurry is not rigorous. For he faults have been described above, we must make at least three experimental observations about the pressure values. If we repeatedly observed that the pressure value is still abnormal, we can check and locate the fault, according to the analysis of the faults above ${ }^{[10]}$.

\section{The pressure detection process of supplying the slurry}

The principle of detecting process of supplying the slurry is same with the pressure detection process of discharging the slurry. Of course, the check and analysis of the

Fault is similar too. It is not too much will be described about it.

\section{Conclusion}

At present, the tailing heap of metal mines are built more and more high and farther. So people have a higher requirement by the transportation of the tailing heap. Because of the need for environmental protection, most of the tailing heap needs to be transported by the slurry pipeline. So the use of the water isolated slurry pump system becomes more common, and we should open and close the high pressure water valve very frequently. It is highly desirable that we should quickly determine whether the high pressure water valve wrong. We can quickly determine and judge the fault point of the high pressure water valve through the method provided in this text.

\section{References}

[1] S.Z. Wang: The slurry pipeline transport of granular materials. Ocean Press -Publishing, Beijing, 1998.1(In china)

[2] X.J. Fei: The transport hydraulics of slurry and granular material. Tsinghua University PressPublishing, Beijing, 1994(In china)

[3] K.M. Zhang, G.A. Li, X.T. Yang: The troubleshooting of isolated pump hydraulic system. Journal of Mining Machinery press, (2010) No.5, p.122-124. (In china)

[4]Z.R. Zhang: The vibration analysis and institutional advancement design of high pressure water valve in the water isolation pump. Journal of Machinery design and manufacture press, (2009)No. 3,p.153-155. (In china)

[5]Z.G. Chen, S.K. Meng, S.Y. Cai: The design and research of high pressure water valve in water isolated slurry pump. Journal of Mining press, (2007) No.4, p.70-71. (In china) 
[6]P.Sun, M.X. Jia, Y.B. Tian: The isolation and solutions of water hammer problem in Water Isolated Slurry Pump. Journal of Mining press, (2002) No.7, p.30-54. (In china)

[7]P.Sun, D.Z.Liu: The design and application in the slurry transportation about the isolated slurry pump. Journal of Nonferrous Metals (Mineral Processing) press, (2002) No.6, p.26-27. (In china)

[8] W.Shi: Application of high density tailings pulp transport technology. Journal of Energy Saving of Non-Ferrous Metallurgy press, (2002) Vol.19, No.5, p.9-13. (In china)

[9] P.Sun, Z.Y, W.Y, G.Q.Wang: The hazards and solutions about the water hammer about the slurry pipeline transport system. Journal of General Machinery press, (2003) No.3, p.40-42. (In china)

[10]J.B.Zhang: Analysis and Control on the Noise in Water-Isolation Pump. Journal of Shenyang University press, (2009) No.2, p.105-107. (In china) 